\title{
ON THE MOTION PAST THIN AIRFOILS OF INCOMPRESSIBLE FLUIDS WITH CONDUCTIVITY TENSOR*
}

\author{
BY \\ L. DRAGOS \\ Bucharest University
}

\begin{abstract}
This note is a study of the problem of the steady motion of an inviscid incompressible fluid past thin airfoils, the Hall effect being taken into account. The case of crossed fields (one of the most important in aerodynamics) is studied in detail. The results for the cases of aligned fields and Alfvén motion are also given. The general solution is represented by a continuous superposition of plane waves. The boundary conditions determine the solution by means of a Fredholm-type integral equation which may be solved with the aid of the method of successive approximations. If the parameter of the magneto-hydrodynamic interaction $(S)$ is equal to zero, one obtains the known solution of classical aerodynamics. The equation is solved explicitly for Alfvén motion.

1. Introduction. The flow of conducting incompressible fluids past thin airfoils when the Hall effect is neglected has been rather comprehensively discussed. Relevant references may be found, for instance, in [1] and [2]. In practical problems which require the study of the fluid motion past airfoils (e.g. problems of aerodynamics and astronautics) the fluid is an ionized gas. It is known, however, that in the dynamics of ionized gases the Hall effect cannot be disregarded. Accordingly an examination including the Hall effect is necessary; this is the object of the present paper.

Some results in this connection have already been given. The aligned-fields case has been considered by Sears and Resler [1] and Tang and Seebass [3]. In [1] a qualitative study was made and in [3] a quantitative description of the solution in symmetrical flow was given. Working independently, we examined the general problem in [2] and [4]. In these papers, however, the solution is expressed with the aid of generalized functions.

The solution presented here includes the cases of crossed fields, aligned fields and Alfvén motion. Moreover, the solution is valid for general flows (not only for symmetric flows), and is expressed by means of classical functions. The method is applicable both to compressible fluids [5] and viscous fluids [6].

For the sake of simplicity only the mathematical treatment of the problem is given here. It is assumed that the formulation of the problem is known from the works cited. The notation given in [2] and [4] is used.
\end{abstract}

\section{A. Crossen Fields}

2. Equations of motion. If the free veam velocity and the magnetic induction are orthogonal, then the perturbed motion is determined by the following system of equations [2]:

${ }^{*}$ Received March 13, 1969; revised version received October 10, 1969. 


$$
\begin{gathered}
\frac{\partial v_{x}}{\partial x}+\frac{\partial v_{\nu}}{\partial y}=0, \quad \frac{\partial b_{x}}{\partial x}+\frac{\partial b_{\nu}}{\partial y}=0, \\
\frac{\partial v_{x}}{\partial x}+\frac{\partial p}{\partial x}=S\left(\frac{\partial b_{x}}{\partial y}-\frac{\partial b_{\nu}}{\partial x}\right), \quad \frac{\partial v_{\nu}}{\partial x}+\frac{\partial p}{\partial y}=0, \quad \frac{\partial v_{z}}{\partial x}=S \frac{\partial b_{z}}{\partial y}, \\
R\left(v_{x}+b_{\nu}\right)+\frac{\partial b_{x}}{\partial y}-\frac{\partial b_{\nu}}{\partial x}-\nu \frac{\partial b_{z}}{\partial y}=0, \\
R e_{x}=-\nu R\left(v_{x}+b_{\nu}\right)+R v_{z}+\left(1+\nu^{2}\right) \frac{\partial b_{z}}{\partial y}, \\
R_{\nu}=\left(R-\frac{\partial}{\partial x}\right) b_{z}, \quad \frac{\partial e_{y}}{\partial x}-\frac{\partial e_{x}}{\partial y}=0, \\
\lim _{x^{2}+\nu^{2} \rightarrow \infty}\left(v_{x}, v_{\nu}, v_{z}, p, b_{x}, b_{\nu}, b_{z}, e_{x}, e_{\nu}\right)=0 .
\end{gathered}
$$

From (1) and the first of Eqs. (2) we have

$$
\Delta v_{\nu}+S \Delta b_{x}=0, \quad \Delta=\partial^{2} / \partial x^{2}+\partial^{2} / \partial y^{2} .
$$

From (3) and (4) we eliminate $v_{x}+b_{y}$. From the resulting equation, from (5) and the last of Eqs. (2) we also eliminate $e_{x}, e_{y}$ and $v_{z}$. We obtain

$$
P b_{z}+\nu \frac{\partial}{\partial x} \Delta b_{x}=0, \quad P=\Delta \frac{\partial}{\partial x}+R S \frac{\partial^{2}}{\partial y^{2}}-R \frac{\partial^{2}}{\partial x^{2}} .
$$

By deriving (6) with respect to $x$ and $y$ and taking account of (1), we find an equation in $v_{v}, b_{x}$ and $b_{z}$. On eliminating from this equation $v_{v}$ with the aid of (6) we get

$$
\Delta P b_{x}-\nu \Delta \frac{\partial}{\partial x} \frac{\partial^{2}}{\partial y^{2}} b_{z}=0
$$

so that:

$$
\begin{aligned}
\Delta L\left\{\begin{array}{l}
v_{y} \\
b_{x}
\end{array}\right\}=0, \quad L & =P^{2}+\nu^{2} \frac{\partial^{4}}{\partial x^{2} \partial y^{2}} \Delta ; \\
L b_{z} & =0 .
\end{aligned}
$$

For $\nu=0$ we obtain the equation valid when the Hall effect is negligible [7].

3. Dispersion equation. For plane waves of the form $\exp (-i \lambda x+s y), s=-\mathbf{i} \lambda r$, we obtain the following dispersion equation from (10):

$$
r^{2}+1=0, \quad\left\{i \lambda\left(r^{2}+1\right)-R S r^{2}+R\right\}^{2}=\nu^{2} \lambda^{2} r^{2}\left(r^{2}+1\right) .
$$

The last equation may be also written

$$
\begin{gathered}
a\left(r^{2}+1\right)^{2}+2 b\left(r^{2}+1\right)+c=0, \\
a=(i \lambda-R S)^{2}-\nu^{2} \lambda^{2}=a_{1}+i a_{2}, \quad c=R^{2}(S+1)^{2}, \\
b=R(S+1)(i \lambda-R S)+\frac{1}{2} \nu^{2} \lambda^{2}=b_{1}+i b_{2} .
\end{gathered}
$$

The roots of Eq. (12) are distinct and complex. The first assertion results from the relation $b^{2} \not \equiv a c$ and the second from the fact that if in (12) $r$ had been real, we should have had

$$
a_{1}\left(r^{2}+1\right)^{2}+2 b_{1}\left(r^{2}+1\right)+c=0, \quad a_{2}\left(r^{2}+1\right)+2 b_{2}=0 .
$$


But the real roots of the last equation, $S r^{2}=1$, do not verify identically the previous equation.

We denote $r_{0}=-i \operatorname{sign} \lambda$ and by $r_{i}(j=1,2)$ the roots of Eq. (12) for which $s_{i}=$ $-i \lambda r_{i}$ have negative real parts. Since Eq. (12) is biquadratic, two roots have this property no matter whether $\lambda$ is positive or negative. The last assertion results from the fact that the roots of Eq. (12) are expressed by radicals and from the observation that for two real numbers $\alpha$ and $\beta$ we have

$$
\left(2 \alpha+2 i \beta \lambda^{-1}\right)^{1 / 2}=\operatorname{sign} \beta\left\{\left(\alpha^{2}+\beta^{2} \lambda^{-2}\right)^{1 / 2}+\alpha\right\}^{1 / 2}+i \operatorname{sign} \lambda\left\{\left(\alpha^{2}+\beta^{2} \lambda^{-2}\right)^{1 / 2}-\alpha\right\}^{1 / 2} .
$$

The remaining three roots have positive real part.

Elementary calculations show that for large $\lambda$ we have

$$
\begin{aligned}
r_{1} & =-i \operatorname{sign} \lambda+O\left(R^{2} \lambda^{-2}\right), \\
\left(1+\nu^{2}\right)^{1 / 2} r_{2} & =-i \operatorname{sign} \lambda+\left\{1+S\left(1+\nu^{2}\right)^{-1}\right\} R|\lambda|^{-1}+O\left(R^{2} \lambda^{-2}\right) .
\end{aligned}
$$

4. General solution. Taking account of $(6),(10)$ and $\left(10^{\prime}\right)$ we have

$$
\begin{gathered}
\left\{\begin{array}{c}
v_{y}^{ \pm} \\
b_{x}^{ \pm}
\end{array}\right\}(x, y)=\mp \int_{-\infty}^{+\infty} \sum_{k=0,1,2} r_{k}\left\{\begin{array}{l}
A_{k}^{ \pm} \\
B_{k}^{ \pm}
\end{array}\right\} \exp \left(-i \lambda x \pm s_{k} y\right) d \lambda ; \\
\nu b_{z}^{ \pm}(x, y)=\int_{-\infty}^{+\infty} \sum_{i=1,2} C_{i}^{ \pm} \exp \left(-i \lambda x \pm s_{i} y\right) d \lambda .
\end{gathered}
$$

From (1) and the last of Eqs. (2) we also have

$$
\begin{gathered}
\left\{\begin{array}{l}
v_{x}^{ \pm} \\
b_{y}^{ \pm} \\
p^{ \pm}
\end{array}\right\}(x, y)=\int_{-\infty}^{+\infty} \sum_{k}\left\{\begin{array}{c}
r_{k}^{2} A_{k}^{ \pm} \\
B_{k}^{ \pm} \\
A_{k}^{ \pm}
\end{array}\right\} \exp \left(-i \lambda x \pm s_{k} y\right) d y ; \\
v v_{z}^{ \pm}(x, y)= \pm S \int_{-\infty}^{+\infty} \sum_{i} r_{i} C_{i}^{ \pm} \exp \left(-i \lambda x \pm s_{i} y\right) d \lambda .
\end{gathered}
$$

Taking account of (11), the first of Eqs. (2) and Eq. (3) yield

$$
\begin{gathered}
A_{i}^{ \pm}+S B_{i}^{ \pm}=0, \quad A_{0}^{ \pm}=B_{0}^{ \pm}, \\
s_{i} C_{i}^{ \pm}= \pm P_{i} B_{i}^{ \pm}, \quad P_{j}=i \lambda\left(r_{i}^{2}+1\right)-R S r_{i}^{2}+R .
\end{gathered}
$$

Finally, from (4) and (5) we get

$$
\nu R\left\{\begin{array}{c}
e_{x}^{ \pm} \\
e_{y}^{ \pm}
\end{array}\right\}(x, y)=\int_{-\infty}^{+\infty}\left(R i \lambda^{-1}-1\right) \sum_{i}\left\{\begin{array}{c}
r_{i}^{-1} \\
\pm 1
\end{array}\right\} r_{i}^{-1} P_{i} B_{i}^{ \pm} \exp \left(-i \lambda x \pm s_{i} y\right) d \lambda .
$$

In the above notation the top line indicates the solution valid in the upper half-plane $(y>0)$ and the bottom line the solution in the lower half-plane $(y<0)$.

The unknowns $B_{k}^{ \pm}$are determined from the boundary conditions.

5. Boundary conditions. We assume that the airfoil equation is

$$
y=Y_{ \pm}(x), \quad|x| \leq 1,
$$

$Y_{ \pm}^{\prime}$ satisfying Hölder's condition. The following relations result: 


$$
\begin{gathered}
v_{\nu}^{+}(x, 0)=Y_{+}^{\prime}(x), \quad|x| \leq 1, \\
{\left[v_{\nu}\right]=\epsilon(x)\left[Y^{\prime}\right], \quad\left[b_{x}\right]=0, \quad \forall x,} \\
{\left[b_{z}\right]=\left[b_{\nu}\right]=\left[e_{x}\right]=0, \quad \forall x,}
\end{gathered}
$$

where $[\phi]=\phi^{+}(x, 0)-\phi^{-}(x, 0)$ and

$$
\begin{aligned}
\epsilon(x) & =1 & & |x| \leq 1, \\
& =0 & & |x|>1 .
\end{aligned}
$$

By using the inversion theorem of Fourier transforms, we deduce the following equations from the general solution and the boundary conditions:

$$
\begin{gathered}
\int_{-\infty}^{+\infty}\left(-r_{0} B_{0}^{+}+S \sum r_{i} B_{i}^{+}\right) \exp (-i \lambda x) d \lambda=Y_{+}^{\prime}(x), \quad|x| \leq 1, \\
r_{0}\left(B_{0}^{+}+B_{0}^{-}\right)-S \sum r_{i}\left(B_{i}^{+}+B_{i}^{-}\right)=2 I, \\
\sum r_{k}\left(B_{k}^{+}+B_{k}^{-}\right)=0, \quad \sum r_{i}^{-1} P_{j}\left(B_{i}^{+}+B_{i}^{-}\right)=0 \\
2 I=-\frac{1}{2 \pi} \int_{-1}^{+1}\left[Y^{\prime}\right] \exp (i \lambda x) d \lambda, \\
\sum\left(B_{k}^{+}-B_{k}^{-}\right)=0, \quad \sum r_{j}^{-2} P_{i}\left(B_{i}^{+}-B_{i}^{-}\right)=0 .
\end{gathered}
$$

From (24) we get

$$
\begin{gathered}
B_{k}^{+}+B_{k}^{-}=2 \alpha_{k} I, \\
(S+1) \alpha \alpha_{1}=-r_{1} P_{2}, \quad(S+1) \alpha \alpha_{2}=r_{2} P_{1}, \\
(S+1) \alpha_{\iota} r_{0}=1, \quad \alpha=(i \lambda+R)\left(r_{1}^{2}-r_{2}^{2}\right) .
\end{gathered}
$$

Since Eqs. (25) become

there results

$$
\sum B_{k}^{+}=\left(\sum \alpha_{k}\right) I, \quad \sum r_{i}^{-2} P_{j} B_{i}^{+}=\left(\sum r_{i}^{-2} P_{j} \alpha_{j}\right) I,
$$

$$
\begin{aligned}
& B_{i}^{+}=\beta_{j} B_{0}^{+}+\gamma_{i} I, \\
& \beta_{1}=-r_{1}^{2} P_{2} \alpha^{-1}, \quad \gamma_{1}=\alpha_{1}+\alpha_{0} r_{1}^{2} P_{2} \alpha^{-1} \text {, } \\
& \beta_{2}=r_{2}^{2} P_{1} \alpha^{-1}, \quad \gamma_{2}=\alpha_{2}-\alpha_{0} r_{2}^{2} P_{1} \alpha^{-1} .
\end{aligned}
$$

Taking account of (27), Eq. (23) becomes

$$
\begin{aligned}
\int_{-\infty}^{+\infty}\left(r_{0}-S \sum r_{i} \beta_{i}\right) B_{0}^{+} & \exp (-i \lambda x) d \lambda \\
& =S \int_{-\infty}^{+\infty} I\left(\sum r_{i} \gamma_{i}\right) \exp (-i \lambda x) d \lambda-Y_{+}^{\prime}, \quad|x| \leq 1 .
\end{aligned}
$$

On using the pressure expression we deduce

$$
\begin{aligned}
{[p]=\int_{-\infty}^{+\infty}\left\{B_{0}^{+}-B_{0}^{-}-S\right.} & \left.\sum\left(B_{i}^{+}-D_{i}^{-}\right)\right\} \exp (-i \lambda x) d \lambda \\
& =2(S+1) \int_{-\infty}^{+\infty}\left(B_{0}^{+}-\alpha_{0} I\right) \exp (-i \lambda x) d \lambda=2 f(x),
\end{aligned}
$$

which is useful for the calculation of lift. 
The condition of pressure continuity outside the airfoil yields

$$
f(x) \equiv 0, \quad|x|>1 \text {. }
$$

By means of the inversion formula for Fourier integrals, we deduce from (29)

$$
B_{0}^{+}=\alpha_{0} I+\frac{1}{2 \pi(S+1)} \int_{-1}^{+1} f(t) \exp (i \lambda t) d t,
$$

so that (28) becomes

$$
\begin{gathered}
\int_{-1}^{+1} f(t) K(t-x) d t=(S+1) Y(x), \quad|x| \leq 1, \\
\left.K(t-x)=-\frac{1}{2 \pi} \int_{-\infty}^{+\infty} k \exp \{i \lambda(t-x)\} d \lambda, \quad Y=\frac{1}{2}: Y_{+}^{\prime}+Y_{-}^{\prime}\right), \\
k=r_{0}+S\left(r_{1}+r_{2}\right)^{-1}\left\{r_{1}^{2}+r_{1} r_{2}+r_{2}^{2}+r_{1}^{2} r_{2}^{2}(i \lambda-R S)(i \lambda+R)^{-1}\right\} .
\end{gathered}
$$

In conclusion, by determining $f(x)$ from Eq. (32) we get from (31) $B_{0}^{+}$, from (27) $B_{i}^{+}$and from (26) $B_{k}^{-}$. The lift may be calculated with the aid of (29). All the determinations can be made since the roots $r_{i}$ are distinct.

6. The Fredholm-type equation. Taking into account (14) we obtain

$$
\begin{aligned}
& k=-(S+1) i \operatorname{sign} \lambda-S(S+1) k^{*}, \\
& k^{*}=\frac{\omega}{|\lambda|}+O\left(R^{2} \lambda^{-2}\right), \quad \omega=\frac{R}{1+\nu^{2}+\left(1+\nu^{2}\right)^{1 / 2}},
\end{aligned}
$$

which shows that the kernel (32') is a distribution. According to [8],

$$
\int_{-\infty}^{+\infty} \operatorname{sign} \lambda \exp \{i \lambda(t-x)\} d t=2 i(t-x)^{-1},
$$

so that there results

$$
K(t-x)=-\frac{S+1}{\pi(t-x)}-S(S+1) K^{*}(t-x),
$$

$K^{*}$ being obtained from the expression of $K$ by replacing $k$ by $k^{*} . K^{*}$ is a convergent integral.

Accordingly, Eq. (32) becomes the singular integral equation

$$
\frac{1}{\pi} \rho \int_{-1}^{+1} \frac{f(t)}{t-x} d t+S \int_{-1}^{+1} f(t) K^{*}(t-x) d t=-Y(x), \quad|x| \leq 1,
$$

where the sign " $\odot$ " indicates the principal value in the sense given by Cauchy.

In the aerodynamics of thin airfoils the singular integral equation

$$
\frac{1}{\pi} \odot \int_{-1}^{+1} \frac{f(t)}{t-x} d t=F(x), \quad|x| \leq 1
$$

occuis. ', 'c solution of this equation, assuming that $F$ satisfies Hölder's condition, is, from [9] and [10],

$$
f(x)=-\frac{1}{\pi}\left(\frac{1-x}{1+x}\right)^{1 / 2} \odot \int_{-1}^{+1}\left(\frac{1+t}{1-t}\right)^{1 / 2} \frac{F(t)}{t-x} d t .
$$


With this result, we obtain from (36) the following Fredholm-type equation:

$$
\begin{aligned}
f(x) & =S \int_{-1}^{+1} f(\xi) M(\xi, x) d \xi+m(x) \\
M(\xi, x) & =\frac{1}{\pi}\left(\frac{1-x}{1+x}\right)^{1 / 2} \odot \int_{-1}^{+1}\left(\frac{1+t}{1-t}\right)^{1 / 2} \frac{K^{*}(\xi-t)}{t-x} d t, \\
m(x) & =\frac{1}{2 \pi}\left(\frac{1-x}{1+x}\right)^{1 / 2} \odot \int_{-1}^{+1}\left(\frac{1+t}{1-t}\right)^{1 / 2} \frac{Y_{+}^{\prime}(t)+Y_{-}^{\prime}(t)}{t-x} d t .
\end{aligned}
$$

The integral equation (38) solves the problem completely. It may be integrated by the method of successive approximations, the first approximation being the solution given in classical aerodynamics $(S=0)$. The solution is general. By particularization we deduce the solution given by Sears and Resler [11] for the perfectly conducting fluid as well as the solution for the fluid with nonzero electrical resistivity [12], [13], [7].

\section{B. Aligned Fields}

7. Motion equation and the general solution. In this case the disturbed motion is determined from the following system (see [2] and [4]):

$$
\begin{gathered}
\frac{\partial v_{x}}{\partial y}-\frac{\partial v_{y}}{\partial x}+S\left(\frac{\partial b_{y}}{\partial x}-\frac{\partial b_{x}}{\partial y}\right)=0, \quad v_{x}=-p, \quad v_{z}=S b_{z}, \\
R\left(v_{\nu}-b_{\nu}\right)-\frac{\partial b_{x}}{\partial y}+\frac{\partial b_{y}}{\partial x}+\nu \frac{\partial b_{z}}{\partial x}=0, \\
R e_{x}=\frac{\partial b_{z}}{\partial y}, \quad R e_{\nu}=\nu\left(\frac{\partial b_{x}}{\partial y}-\frac{\partial b_{y}}{\partial x}\right)-\frac{\partial b_{z}}{\partial x}+R(1-S) b_{z},
\end{gathered}
$$

to which we add Eqs. (1), (6) and the last of Eqgs. (.5). From the above system of equations we get

$$
\begin{gathered}
L b_{z}=0, \quad \Delta I\left\{\begin{array}{l}
{ }^{\prime \prime} u \\
b_{u}
\end{array}\right\}=0, \\
L=P^{2}+\nu^{2} \Delta \partial^{2} / \partial x^{2}, \quad I=\Delta+R(S-1) \partial / \partial x .
\end{gathered}
$$

For plane waves of the same type as in the preceding paragraph we have the following dispersion equations:

$$
r^{2}+1=0, \quad\left\{r^{2}+1+i \lambda^{-1} R(S-1)\right\}^{2}+\nu^{2}\left(r^{2}+1\right)=0 .
$$

Denoting by $r_{i}(j=1,2)$ the roots of Eq. (43) for which $s_{i}=-i \lambda r_{i}$ have negative real parts, we deduce

$$
\begin{aligned}
& r_{1}=-i \operatorname{sign} \lambda+R^{2}(S-1)^{2} O\left(\lambda^{-2}\right), \\
& r_{2}=-i\left(1+\nu^{2}\right)^{1 / 2} \operatorname{sign} \lambda+\frac{R(S-1)}{\left(1+\nu^{2}\right)^{1 / \sqrt{2}}} \frac{1}{|\lambda|}+R^{2}(S-1)^{2} O\left(\lambda^{-2}\right) .
\end{aligned}
$$

We also set $r_{0}=-i \operatorname{sign} \lambda$.

Taking account of the damping condition (6) we get the following general solution: 


$$
\begin{gathered}
\left\{\begin{array}{l}
v_{y}^{ \pm} \\
v_{x}^{ \pm} \\
b_{y}^{ \pm} \\
b_{x}^{ \pm}
\end{array}\right\}(x, y)=\int_{-\infty}^{+\infty} \sum_{k=0,1,2}\left\{\begin{array}{c}
A_{k}^{ \pm} \\
\mp r_{k} A_{k}^{ \pm} \\
B_{k}^{ \pm} \\
\mp r_{k} B_{k}^{ \pm}
\end{array}\right\} \exp \left(-i \lambda x \pm s_{k} y\right) d \lambda, \\
\nu\left\{\begin{array}{c}
b_{z}^{ \pm} \\
R e_{x}^{ \pm}
\end{array}\right\}(x, y)=\int_{-\infty}^{+\infty} \sum_{i=1,2}\left\{\begin{array}{c}
C_{i}^{ \pm} \\
\pm s_{i} C_{i}^{ \pm}
\end{array}\right\} \exp \left(-i \lambda x \pm s_{i} y\right) d \lambda, \\
A_{i}^{ \pm}=S B_{i}^{ \pm}(S \neq 1), \quad \begin{array}{c}
A_{0}^{ \pm}=B_{0}^{ \pm}, \\
C_{i}^{ \pm}=-P_{j} B_{i}^{ \pm}, \quad P_{j}=r_{i}^{2}+1+i \lambda^{-1} R(S-1) .
\end{array}
\end{gathered}
$$

As the notations coincide with those used in the preceding paragraph, they do not lead to ambiguities.

8. Boundary conditions. Conditions $(20)-(22)$ furnish the equations:

$$
\begin{gathered}
\int_{-\infty}^{+\infty}\left(B_{0}^{+}+S B_{1}^{+}+S B_{2}^{+}\right) \exp (-i \lambda x) d \lambda=Y_{+}^{\prime}(x), \quad|x| \leq 1, \\
B_{0}^{+}-B_{0}^{-}+S \sum\left(B_{i}^{+}-B_{i}^{-}\right)=-2 I, \\
\sum r_{k}\left(B_{k}^{+}+B_{k}^{-}\right)=0, \quad \sum P_{j}\left(B_{i}^{+}-B_{j}^{-}\right)=0, \\
\sum\left(B_{k}^{+}-B_{k}^{-}\right)=0, \quad \sum r_{j} P_{i}\left(B_{i}^{+}+B_{j}^{-}\right)=0 .
\end{gathered}
$$

We get

$$
\begin{gathered}
B_{k}^{+}-B_{k}^{-}=2 \alpha_{k} I, \\
(S-1) \alpha_{0}=1, \quad(S-1)\left(P_{2}-P_{1}\right) \alpha_{1}=-P_{2}, \quad(S-1)\left(P_{2}-P_{1}\right) \alpha_{2}=P_{1}, \\
B_{i}^{+}=\beta_{i} B_{0}^{+}+\gamma_{j} I, \\
r_{2}\left(P_{2}-P_{1}\right) \beta_{2}=r_{0} P_{1}, \quad(S-1) r_{2}\left(P_{2}-P_{1}\right) \gamma_{2}=\left(r_{2}-r_{0}\right) P_{1}, \\
r_{1}\left(P_{2}-P_{1}\right) \beta_{1}=-r_{1} P_{2}, \quad(S-1) r_{1}\left(P_{2}-P_{1}\right) \gamma_{1}=\left(r_{0}-r_{1}\right) P_{2} .
\end{gathered}
$$

On using the pressure expression we get

$$
[p]=2 \int_{-\infty}^{+\infty}\left\{\left(r_{0}+S \sum r_{i} \beta_{i}\right) B_{0}^{+}+r_{0} I\right\} \exp (-i \lambda x) d \lambda=2 f(x)
$$

Imposing the condition $f(x) \equiv 0,|x|>1$, we get from (51)

$$
(S-1) B_{0}^{+}=I-\frac{1}{2 \pi r_{0}} \int_{-1}^{+1} f(t) \exp (i \lambda t) d t,
$$

so that (47) becomes

$$
\int_{-1}^{+1} f(t) K(t-x) d t=(S-1) Y(x), \quad|x| \leq 1,
$$

$K$ 'ng the same expression (32'), whers:

$$
\begin{aligned}
& k=r_{0}^{-1}-S^{1+r_{1}^{2}+r_{1} r_{1}+r_{2}^{2}+i \lambda^{-1} R(S-1)} \\
& =-(S-1) i \operatorname{sign} \lambda-S(S-1) k^{* *}, \quad k^{* *}=\omega /|\lambda|+O\left(R^{2}(S-1) \lambda^{-2}\right) \text {. }
\end{aligned}
$$


Accordingly, Eq. (53) transforms into the following singular equation:

$$
\frac{1}{\pi} \odot \int_{-1}^{+1} \frac{f(t)}{t-x} d t+S \int_{-1}^{+1} f(t) K^{* *}(t-x) d t=-Y(x), \quad|x| \leq 1 .
$$

With the aid of the method given in the preceding paragraph Eq. (54) reduces to a Fredholm integral equation. Once the function $f$ is determined, formulae (52)-(49) solve the problem. Obviously the above results are valid only if $S \neq 1$.

\section{Alfrén Mótion}

9. General solution. This section examines the singular case $S=1$, the fields being aligned. If in the system of motion equations from Sec. B we set $S=1$, we deduce

$$
\Delta\left(\Delta+\nu^{2} \frac{\partial^{2}}{\partial x^{2}}\right)\left\{\begin{array}{l}
v_{\nu} \\
b_{\nu} \\
b_{z}
\end{array}\right\}=0 .
$$

Since in Eq. (55) there intervene only derivatives of the same order, wave propagation will take place without dispersion. Using the notations:

$$
r_{1}=-i \operatorname{sign} \lambda, \quad r_{2}=-i \mu_{1} \operatorname{sign} \lambda, \quad \mu_{1}=\left(1+\nu^{2}\right)^{1 / 2},
$$

we obtain the following general solution:

$$
\left\{\begin{array}{c}
v_{\nu}^{ \pm} \\
b_{\nu}^{ \pm} \\
\nu b_{z}^{ \pm}
\end{array}\right\}(x, y)=\int_{-\infty}^{+\infty} \sum_{i=1,2}\left\{\begin{array}{c}
A_{i}^{ \pm} \\
B_{i}^{ \pm} \\
C_{i}^{ \pm}
\end{array}\right\} \exp \left(-i \lambda x \pm s_{i} y\right) d \lambda .
$$

From (1), (41), (39), (40) and the last of Eqs. (5) we get successively

$$
\begin{aligned}
& \left\{\begin{array}{c}
v_{x}^{ \pm} \\
b_{x}^{ \pm} \\
\nu R e_{x}^{ \pm}
\end{array}\right\}(x, y)=\int_{-\infty}^{+\infty} \sum_{i}\left\{\begin{array}{l}
\mp r_{i} A_{i}^{ \pm} \\
\mp r_{i} B_{i}^{ \pm} \\
\pm s_{i} C_{i}^{ \pm}
\end{array}\right\} \exp \left(-i \lambda x \pm s_{i} y\right) d \lambda, \\
& A_{2}^{ \pm}=B_{2}^{ \pm}, \quad i \lambda C_{1}^{ \pm}=R\left(A_{1}^{ \pm}-B_{1}^{ \pm}\right), \quad C_{2}^{ \pm}=\nu^{2} B_{2}^{ \pm},
\end{aligned}
$$

the unknowns being $A_{1}^{ \pm}, B_{1}^{ \pm}, B_{2}^{ \pm}$.

The boundary conditions (20)-(22) furnish the following relations:

$$
\begin{gathered}
\int_{-\infty}^{+\infty}\left(A_{1}^{+}+B_{2}^{+}\right) \exp (-i \lambda x) d \lambda=Y_{+}^{\prime}(x), \quad|x| \leq 1, \\
A_{1}^{+}-A_{1}^{-}+B_{2}^{+}-B_{2}^{-}=-2 I, \\
B_{1}^{+}+B_{1}^{-}+\mu_{1}\left(B_{2}^{+}+B_{2}^{-}\right)=0, \\
A_{1}^{+}-A_{1}^{-}-\left(B_{1}^{+}-B_{1}^{-}\right)+\mu_{2}^{-1}\left(B_{2}^{+}-B_{2}^{-}\right)=0, \quad i \lambda \nu^{2} \mu_{2}=R, \\
\sum\left(B_{i}^{+}-B_{i}^{-}\right)=0, \quad A_{1}^{+}+A_{1}^{-}-\left(B_{1}^{+}+B_{1}^{-}\right)+\mu_{1} \mu_{2}^{-1}\left(B_{2}^{+}+B_{2}^{-}\right)=0 .
\end{gathered}
$$

From (60) we get 


$$
\begin{aligned}
A_{1}^{+}-A_{1}^{-} & =-2\left(1+\mu_{2}\right) I, \quad B_{1}^{+}-B_{1}^{-}=-2 \mu_{2} I, \quad B_{2}^{+}-B_{2}^{-}=2 \mu_{2} I, \\
\left(1+\mu_{2}\right) B_{1}^{+} & =\mu_{2} A_{1}^{+}, \quad \mu_{1}\left(1+\mu_{2}\right) B_{2}^{+}=-\mu_{2} A_{1}^{+}+\mu_{2}\left(1+\mu_{2}\right)\left(1-\mu_{1}\right) I .
\end{aligned}
$$

We also have

$$
[p]=2 \int_{-\infty}^{+\infty} r_{1}\left\{\left(1+\mu_{2}\right)^{-1} A_{1}^{+}-I\right\} \exp (-i \lambda x) d \lambda=2 f(x) .
$$

Imposing the condition of pressure continuity outside the airfoil, we get $f(x) \equiv 0,|x|>1$, so that from (62) we have

$$
A_{1}^{+}=\left(1+\mu_{2}\right) I+\frac{1+\mu_{2}}{2 \pi r_{1}} \int_{-1}^{+1} f(t) \exp (i \lambda t) d t .
$$

10. Solution of the integral equation. By using formulae (61) and (63) we get the following integral equation from (59):

$$
\int_{-1}^{+1} f(t) N(t-x) d t=-Y(x), \quad|x| \leq 1
$$

where

$$
\begin{gathered}
N(t-x)=(1 / \pi(t-x))-\omega N_{1}(t-x), \\
N_{1}(t-x)=\frac{1}{2 \pi} \int_{-\infty}^{+\infty} \frac{\exp \{i \lambda(t-x)\}}{|\lambda|} d \lambda=-\frac{1}{\pi}(C+\ln |t-x|),
\end{gathered}
$$

$C$ being Euler's constant ( $=0.577215)$. Here we have used (34). The last integral is also a distribution [8].

Consequently, Eq. (64) will be written:

$$
\frac{1}{\pi} \rho \int_{-1}^{+1} \frac{f(t)}{t-x} d t+\frac{\omega}{\pi} \int_{-1}^{+1} f(t)\{C+\ln |t-x|\} d t=-Y(x) .
$$

If we denote

$$
g(x)=-\frac{1}{\pi} \int_{-1}^{+1} f(t)(C+\ln |t-x|) d t
$$

we deduce

$$
g^{\prime}(x)=\frac{1}{\pi} \int_{-1}^{+1} \frac{f(t)}{t-x} d t
$$

so that the integral equation $\left(64^{\prime}\right)$ becomes

$$
g^{\prime}-\omega g=-Y,
$$

the general solution of which is

$$
g(x)=e^{\omega x}\left(\Gamma_{0}-\int_{0}^{x} Y(\xi) e^{-\omega \xi} d \xi\right)
$$

where $\Gamma_{0}$ is a constant.

From (68) we determine the solution of Eq. $\left(64^{\prime}\right)$ 


$$
\begin{aligned}
f(x) & =-\frac{1}{\pi}\left(\frac{1-x}{1+x}\right)^{1 / 2} \odot \int_{-1}^{+1}\left(\frac{1+t}{1-t}\right)^{1 / 2} \frac{g^{\prime}(t)}{t-x} d t=\Gamma_{0} f_{0}(x)+f_{1}(x), \\
f_{1}(x) & =\frac{1}{\pi}\left(\frac{1-x}{1+x}\right)^{1 / 2} \odot \int_{-1}^{+1}\left(\frac{1+t}{1-t}\right)^{1 / 2} \frac{Y^{*}(t)}{t-x} d t \\
Y^{*}(t) & =Y(t)+\omega \int_{0}^{t} Y(\xi) e^{\omega(t-\xi)} d \xi \\
f_{0}(x) & =-\frac{\omega}{\pi}\left(\frac{1-x}{1+x}\right)^{1 / 2} \odot \int_{-1}^{+1}\left(\frac{1+t}{1-t}\right)^{1 / 2} \frac{\exp (\omega t)}{t-x} d t .
\end{aligned}
$$

The last integral can be calculated explicitly by using the known formulae:

$$
\begin{gathered}
\exp (\omega \cos \tau)=I_{0}(\omega)+2 \sum_{1}^{\infty} I_{n}(\omega) \cos n \tau, \\
\frac{1}{\pi} \int_{0}^{\pi} \frac{\cos n \tau}{\cos \tau-\cos \theta} d \tau=\frac{\sin n \theta}{\sin \theta}, \quad n=0,1,2, \cdots,
\end{gathered}
$$

$I_{n}(\omega)$ being the modified Bessel functions.

11. Determination of the lift. The lift is determined by the formula:

$$
L=-\int_{-1}^{+1}[p] d x=-2 \int_{-1}^{+1} f(x) d x,
$$

so that using the solution (70) as well as the formulae (71) and (72) we obtain

$$
L=2 \int_{-1}^{+1}\left(\frac{1+t}{1-t}\right)^{1 / 2} g^{\prime}(t) d t=2 \Gamma_{0} f_{0}^{*}-2 f_{1}^{*}
$$

where

$$
f_{1}^{*}=\int_{-1}^{+1}\left(\frac{1+t}{1-t}\right)^{1 / 2} Y^{*}(t) d t, \quad f_{0}^{*}=\pi \omega\left(I_{0}(\omega)+I_{1}(\omega)\right) .
$$

In order to determine the constant $\Gamma_{0}$ we will use (67). As we have

$$
\begin{aligned}
\int_{-1}^{+1}\left(\frac{1+x}{1-x}\right)^{1 / 2} \ln |t-x| & d x \\
& =\int_{-1}^{+1} \frac{\ln |t-x|}{\left(1-x^{2}\right)^{1 / 2}} d x+\int_{-1}^{+1} \frac{x \ln |t-x|}{\left(1-x^{2}\right)^{1 / 2}} d x=-\pi(\ln 2+t),
\end{aligned}
$$

it follows:

$$
\int_{-1}^{+1}\left(\frac{1+x}{1-x}\right)^{1 / 2} g(x) d x=(\ln 2-C) \int_{-1}^{+1} f(t) d t+\int_{-1}^{+1} f(t) t d t .
$$

By using (70) and (72) (or directly (68)) we deduce

$$
\int_{-1}^{+1} f(t) t d t=\int_{-1}^{+1}\left(1-t^{2}\right)^{1 / 2} g^{\prime}(t) d t=\int_{-1}^{+1}\left(1-t^{2}\right)^{1 / 2}(\omega g-Y) d t
$$

Taking into account $\left(69^{\prime}\right)$, we get from (75) and (76) 
$\pi \Gamma \Gamma_{0}=(\ln 2-C) f_{1}^{*}$

$$
-\int_{-1}^{+1}\left(1-t^{2}\right)^{1 / 2} Y^{*}(t) d t+\int_{-1}^{+1}\left(\frac{1+t}{1-t}\right)^{1 / 2}\left(\int_{0}^{t} Y(\xi) e^{\omega(t-\xi)} d \xi\right) d t
$$

$\Gamma=I_{0}-I_{1}+\omega(\ln 2-C)\left(I_{0}+I_{1}\right)$.

This formula will determine the constant $\Gamma_{0}$ as well as the lift and the solution of the problem.

12. The flat plate. In order to illustrate the above results, we will consider a flat plate at incidence $\epsilon^{*}$. Using the notation $\epsilon=\operatorname{tg} \epsilon^{*}$ we deduce:

$$
\begin{aligned}
Y(t) & =-\epsilon, \quad Y^{*}(t)=-\epsilon \exp (\omega t), \quad f_{1}^{*}=-\epsilon \pi\left(I_{0}+I_{1}\right) \\
\Gamma_{0} & =-\epsilon \omega^{-1}\left(1-\Gamma^{-1}\right), \quad f(x)=\epsilon \omega^{-1} \Gamma^{-1} f_{0}(x) \\
L & =2 \pi \epsilon \Gamma^{-1}\left\{I_{0}(\omega)+I_{1}(\omega)\right\} .
\end{aligned}
$$

The graphs shown in Fig. 1 show both the influence exerted by the fluid resistivity upon the lift, and the influence of the Hall effect. For $R=0$ (nonconductive fluid) the lift reduces to $2 \pi \epsilon$, a known formula from conventional aerodynamics. In fact, in this case

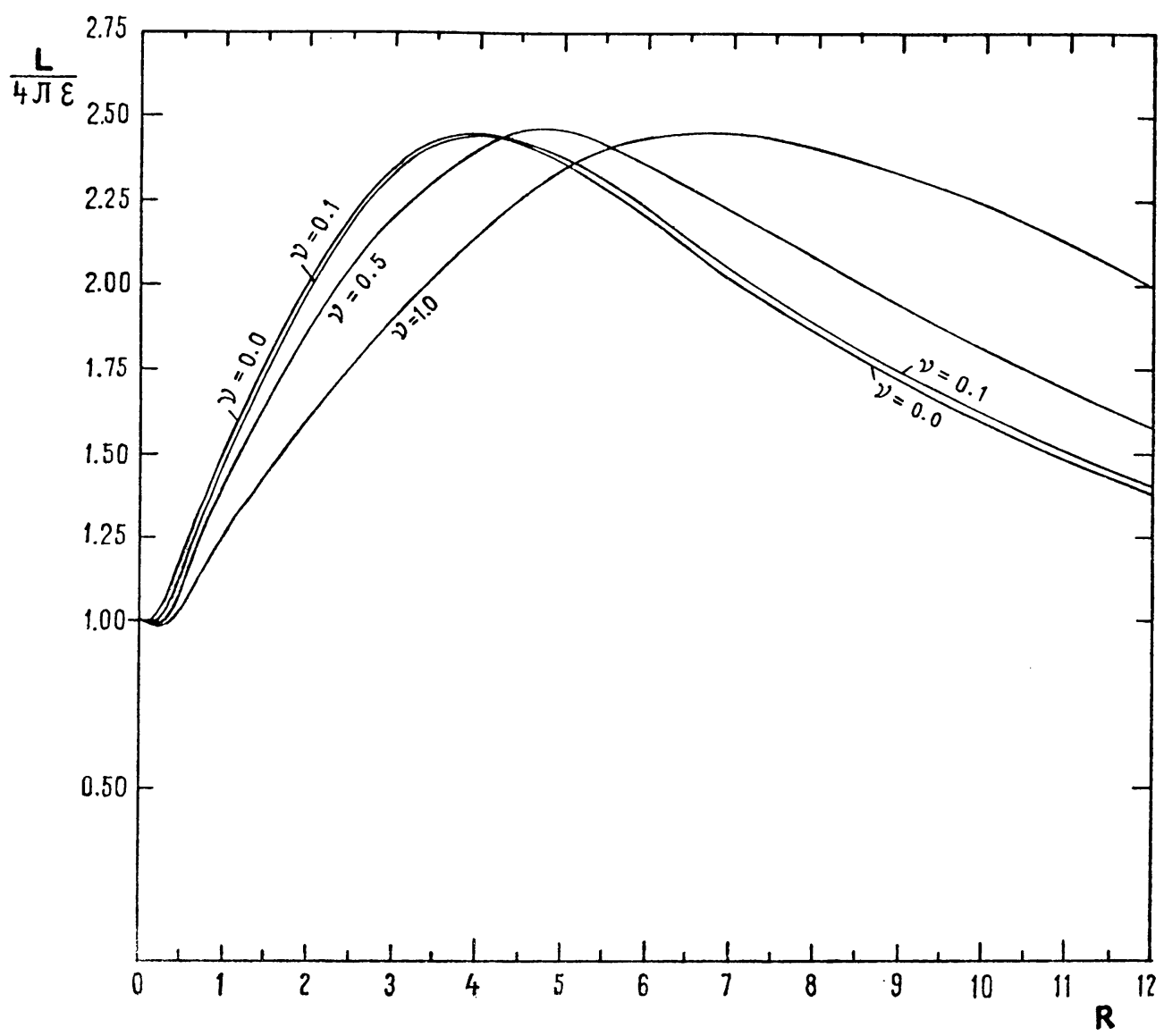

FIG. 1 
the solution (70) also coincides with the solution given in nonconductive aerodynamics. As $R$ increases, the lift begins by decreasing, since

$$
\left.\frac{d L}{d R}\right|_{R=0}=-\frac{\ln 2-C}{1+\nu^{2}+\left(1+\nu^{2}\right)^{1 / 2}}<0 ;
$$

then it increases, becoming approximately two and a half times higher than in the case of nonconductive fluids. This maximum value is reached at $R=4$, when the Hall effect is negligible. As soon as the Hall effect appears the lift becomes maximum for higher values of $R$. After reaching the maximum value, the lift decreases as the conductivity of the fluid increases and tends towards zero for $R \rightarrow \infty$ (for $R=20$ we have $L(2 \pi \epsilon)^{-1}=0.84(\nu=0.0)$, $0.85(\nu=0.1), 1.11(\nu=0.5), 1.39(\nu=1.0))$.

The most important conclusion is that this maximum value of the lift exists.

\section{An Approximate Solution in the General Case}

13. In the case of aligned fields, for lower values of the parameter $R|S-1|$ the expression of the kernel $k\left(53^{\prime}\right)$ can be used in the form:

$$
k=-i(S-1) \operatorname{sign} \lambda-S(S-1) \omega /|\lambda|,
$$

so that the integral equation (54) becomes

$$
\frac{1}{\pi} \odot \int_{-1}^{+1} \frac{f(t)}{t-x} d t+\frac{\omega S}{\pi} \int_{-1}^{+1} f(t)(C+\ln |t-x|) d t=-Y(x) .
$$

In the case of crossed fields, for low values of the parameter $R$, the expression of $k$ given in $\left(32^{\prime}\right)$ becomes:

$$
k=-i(S+1) \operatorname{sign} \lambda-S(S+1) \omega /|\lambda|,
$$

so that the integral equation (36) also reduces to (79).

Eq. (79) coincides with $\left(64^{\prime}\right)$ so that its solution will be given by formulae (70) and (77) in which $\omega$ is substituted for $S \omega$. In particular, the lift will be determined by the formula:

$$
L=2\left(\tilde{\Gamma}_{0} \tilde{f}_{0}^{*}-\tilde{f}_{1}^{*}\right),
$$

the expressions of $\tilde{f}_{0}^{*}, \tilde{f}_{1}^{*}$ and $\tilde{\Gamma}_{0}$ being given by the corresponding formulae given in Sec. 11 with the same substitution effected $(\omega \rightarrow S \omega)$.

The most important conclusion derives from the fact that in this approximation the lift is independent of the orientation of the external magnetic fields; this is acceptable from physical point of view.

\section{REFERENCES}

[1] W. R. Sears and E. L. Resler, Jr., Magneto-hydrodynamic flow past bodies, Advances in Appl. Mech., vol. 8, Academic Press, New York, 1964, pp. 1-68

[2] L. Dragos, Magnetodynamics of fluids, Publ. House Romanian Academy of Science, Bucharest, 1969

[3] J. Y. T. Tang and H. Seebass, The effect of tensor conductivity on continuum magnetodynamic flows, Quart. Appl. Na:h. 26, 311-320 (1968)

[4] L. Dragos, L'effet Hall dans l'écoulement des fluides en présence des profils minces, C. R. Acad. Sci. Paris Ser. A-B 267, A579 (1968)

[5] L. Dragos, L'effet Hall dans l'écoulement des fluides compressibles en présence des profils minces, C. R. Acad. Sci. Paris Ser. A-B 269 (1969) 
[6] L. Dragos, The Hall effect on the motion of viscous fluids past the flat place, Rev. Roumain Math. Pures Appl. 15, (1970)

[7] L. Dragos, Theory of thin airfoils in magnetohydrodynamics, Arch. Rational Mech. Anal. 13, 262278 (1963)

[8] I. M. Gel'fand and G. E. Šilov, Genralized functions. I: Operations on them, Fizmatgiz, Moscow, 1958; English transl., Academic Press, New York, 1964

[9] C. Iacob, Introduction mathématique à la mécanique des fluides, Akad. Repub. Pop. Roumaine, Bucarest and Gauthier-Villars, Paris, 1959

[10] N. I. Mushelišvili, Singular integral equations, OGIZ, Moscow, 1946; English transl., Noordhoff, Groningen, 1953

[11] W. R. Sears and E. J. Resler, Jr., Theory of thin airfoils in fluids of high electrical conductivity, J. Fluid Mech. 5, 257-273 (1959)

[12] K. Stewartson, Magneto-fluid dynamics of thin bodies in oblique fields. II, Z. Angew. Math. Phys. 13, 242-255 (1962)

[13] L. Dragos, La théorie de l'aile mince en magnétohydrodynamique. I, II, C. R. Acad. Sci. Paris 265, $1251-1253 ; 1289-1290$ (1962) 